\title{
The Application of the Selected Time-Frequency Descriptors Detection of the Acoustic Emission Signals Generated by Multisource Partial Discharges
}

\author{
A. CiCHON* \\ Faculty of Electrical Engineering, Automatic Control and Computer Science, Opole University of Technology \\ Mikołajczyka 5, 45-271 Opole, Poland
}

The paper presents the comparative analysis results of the acoustic emission signals generated by various forms of partial discharges occurring in multisource systems. Modern methods of the signal digital processing: a short-time Fourier transform and continuous and discrete wavelet transforms were applied for the assessment of the results in the time-frequency domain. The acoustic emission signals generated by partial discharges were registered with a WD AH17 contact transducer by the Physical Acoustics Corporation placed on the external part of the tub.

PACS numbers: 52.80.-s, 43.28.Js

\section{Introduction}

Insulation diagnostics of high-voltage power appliances by the acoustic emission (AE) method is based on the analysis of the time run of the acoustic wave generated by partial discharges (PDs) which is registered by a piezoelectric sensor. PDs occurring in power transformers are formed at the point of junction of three phases: a copper wire of which a winding is made, a solid dielectric and insulation oil.

In recent years the development of the $\mathrm{AE}$ method was caused by introduction into the description of the AE signals generated by PDs a combined time-frequency analysis. Based on the frequency and time-frequency descriptions it is possible to detect PDs in single-source systems [1-4]. In electric power transformers being in use the occurrence of multi-source discharges is possible. Therefore it is necessary to carry out laboratory research tests on the possibilities of applying the AE method for detection of PDs in multi-source systems. The paper presents an exception from the research work on comparing the $\mathrm{AE}$ signals generated by single- and multi-source PDs.

\section{Measuring setup}

Figure 1 presents a diagram of the measuring system for generation, registration and analysis of the AE signals generated by PDs.

Due to the complexity of the generation processes of the AE signals from multi-source PDs of various types,

* e-mail: a.cichon@po.opole.pl

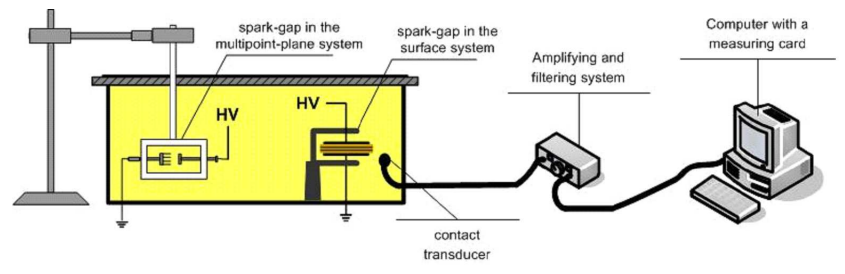

Fig. 1. Diagram of the measuring system.

the research experiment was carried out in the Laboratory of Insulation System Diagnostics of the Electrical Power Engineering of Opole Technical University. The measuring tub was filled with insulation oil, in which two spark-gaps generating PDs were immersed. One spark-gap generated PDs in the surface system and the other generated discharges in the multipoint-plane system. The spark-gaps were supplied from two TP 60 test transformers with voltage corresponding to $80 \%$ of breakdown voltage of each of the systems. The AE signals generated by PDs were registered with a WD AH17 contact transducer by the firm Physical Acoustics Corporation (PAC), which was placed on the external part of the tub. This transducer is characteristic of high sensitivity ( $55 \mathrm{~dB} \pm 1.5 \mathrm{~dB}$ in relation to $\mathrm{Vs} / \mathrm{m}$ ) and a wide transfer band from $100 \mathrm{kHz}$ to $1 \mathrm{MHz}$ in the range $\pm 10 \mathrm{~dB}$.

The measuring transducer was connected with the amplifying and filtering system through a subamplifier. A band-pass filter of cut-off frequencies 10 and $700 \mathrm{kHz}$ was used. The measuring signal was amplified by $43 \mathrm{~dB}$. Time runs of the AE signals generated by PDs were registered with a four-channel measuring card by the firm 
Acquitek CH 3160. During the measurement taking the sampling frequency of $2.56 \mathrm{MHz}$ was applied and the $\mathrm{AE}$ signal of the time of $20 \mathrm{~ms}$ was registered. The testing was carried out in a measuring cell, which was a specialized room; silenced and electromagnetically screened.

\section{Methodology of the research work carried out}

The measurements of the AE signals generated by various PD forms were carried out in model systems, in which three types of spark-gaps were used: point-point, point-plane and surface spark-gaps. The measurement taking consisted in registration of the AE signals generated in multisource systems. Three measurement systems, shown in Figs. 2, 3, 4, were used for this purpose. In each case two spark-gaps were placed in the measurement tub simultaneously and each of them was supplied from a separate transformer with voltage equal to $0.8 U_{\mathrm{p}}$.

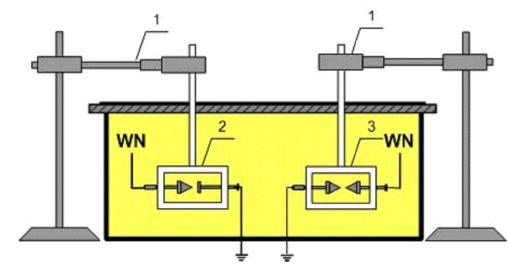

Fig. 2. Diagram of the system for multi-source discharge generation with the use of the point-point and point-plane spark-gaps: 1 - stand, 2 - point-plane spark-gap, 3 - point-point spark-gap.

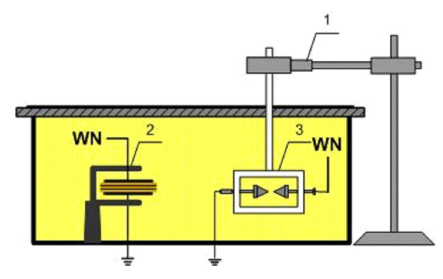

Fig. 3. Diagram of the system for multi-source discharge generation with the use of the point-point and surface spark-gaps: 1 - stand, 2 - surface spark-gap, 3 - point-point spark-gap.

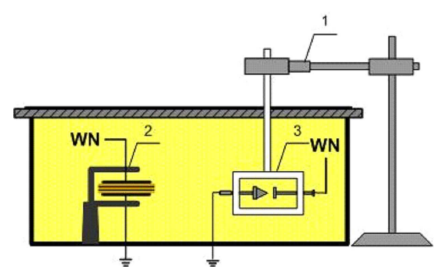

Fig. 4. Diagram of the system for multi-source discharge generation with the use of the point-plane and surface spark-gaps: 1 - stand, 2 - surface spark-gap, 3 - point-plane spark-gap.
The AE signals measured, coming from the particular multi-source PDs, were subjected to the time-frequency analysis using a short-time Fourier transform (STFT) and continuous and discrete wavelet transforms (CWT and DWT). The results obtained were shown using twoand three-dimensional spectrograms of the power spectral density, three-dimensional spectrograms of the amplitude spectrum, CWT scaling graphs and time runs of details at the seventh wavelet decomposition level and column diagrams visualizing the size of the energy are transferred by the particular details.

The wavelet decomposition DWT of the AE pulses measured was carried out by using a symlet wavelet of the eighth order, the time run of which, the scheme of the wavelet decomposition tree structure and the diagram of dependences frequency-scale have been presented, among others, in monograph [1]. Frequency intervals corresponding with the particular details are listed in Table.

TABLE

Width of frequency bands corresponding with the particular details.

\begin{tabular}{c|c|c}
\hline \hline Details & $\begin{array}{c}\text { Median frequency } \\
{[\mathrm{kHz}]}\end{array}$ & $\begin{array}{c}\text { Frequency band } \\
{[\mathrm{kHz}]}\end{array}$ \\
\hline D1 & 500.0 & $250-750$ \\
D2 & 250.0 & $125-375$ \\
D3 & 125.0 & $62.5-187.5$ \\
D4 & 62.5 & $31.2-93.7$ \\
D5 & 31.25 & $15.6-46.8$ \\
D6 & 15.6 & $7.8-23.4$ \\
D7 & 5.86 & $3.91-7.81$
\end{tabular}

The time-frequency analysis was carried out for the AE signals generated by PDs of the length of $20 \mathrm{~ms}$, comprising a whole acoustic event occurring in a single period of the voltage supplying the spark-gap under study.

\section{The analysis of the obtained results}

Figure 5 shows the results of the time-frequency analysis using a short-time Fourier transform (STFT) corresponding with the AE signals generated by $\mathrm{PDs}$ in the multi-source systems.

Figure 6 shows CWT scaling graphs, on which timefrequency pictures of the $\mathrm{AE}$ pulses registered were drawn. The pictures determined by using CWT contain a lot of information of a qualitative character on timefrequency structures of the AE pulses measured.

The results of the multiresolution wavelet decomposition, which were obtained for the AE signals measured, are shown in Fig. 7. Within graphic presentation of the results obtained there are shown, respectively: time run of the AE pulses measured, approximation A at the seventh decomposition level, runs of details D1-D7 and a column diagram visualizing the size of energy transferred by the particular details. 


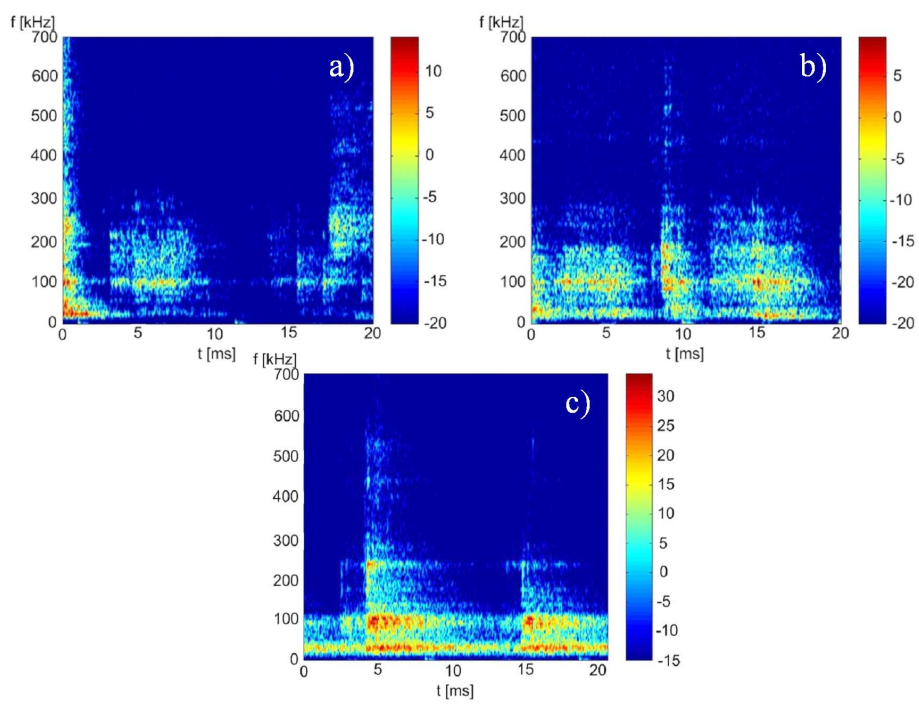

Fig. 5. Two-dimensional spectrograms of power spectral density determined for the $\mathrm{AE}$ signals generated by multi-source PDs with the use of the spark-gaps: (a) point-point and point-plane, (b) point-point and surface, (c) point-plane and surface.

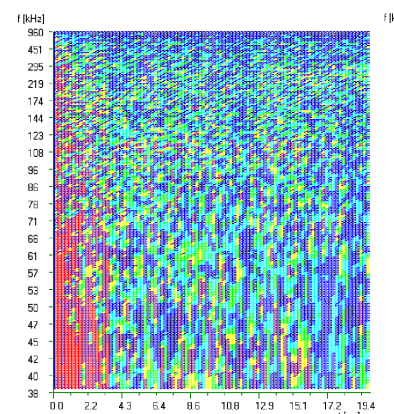

a)

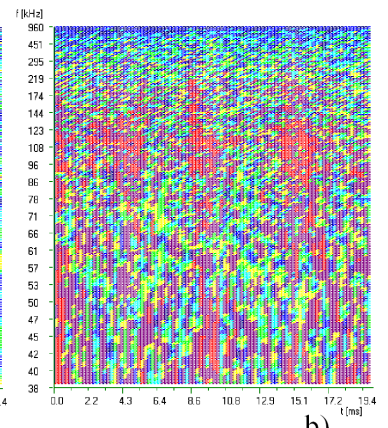

b)

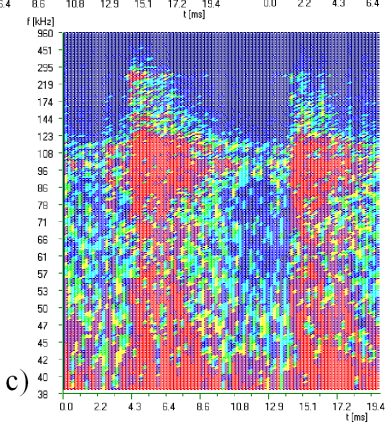

Fig. 6. Continuous wavelet decomposition of the AE signals generated by multi-source PDs with the use of the spark-gaps: (a) point-point and point-plane, (b) point-point and surface, (c) point-plane and surface.

Based on the investigations carried out in single-source systems, the results of which have been presented, among others, in papers [5-10], it was demonstrated that it is possible to assign a characteristic frequency band to the particular discharge forms:

— point-point system $(70-300 \mathrm{kHz})$,

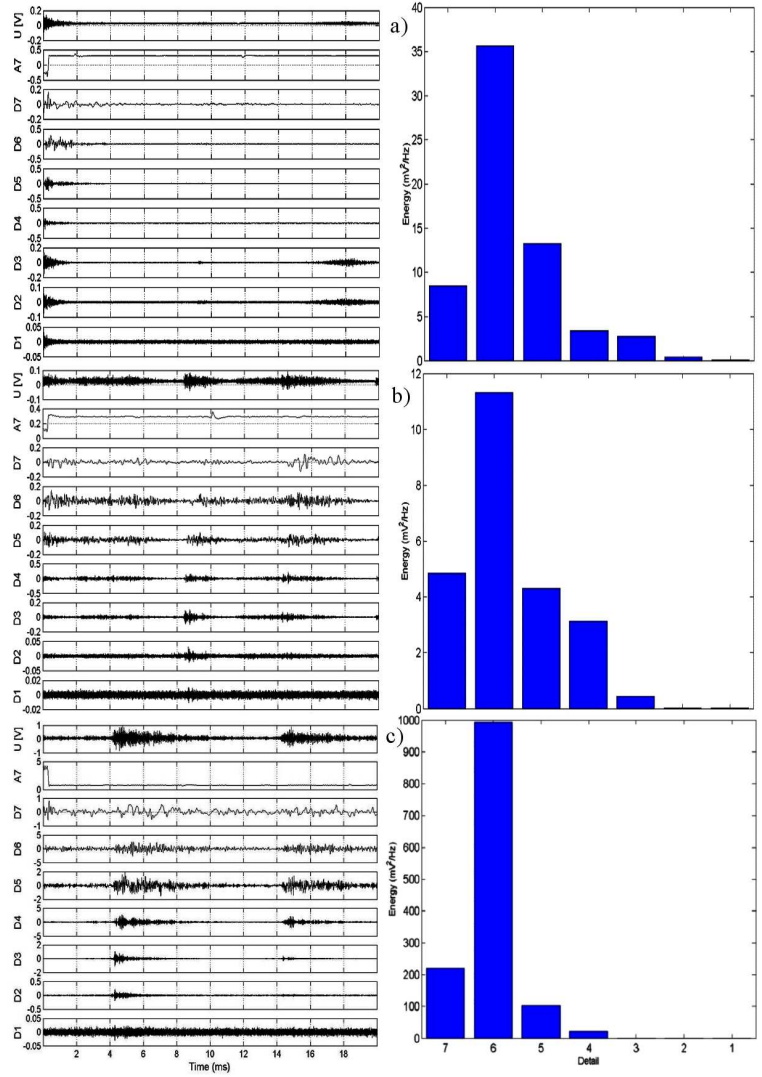

Fig. 7. Discrete wavelet decomposition and energy size transferred by the particular details of the AE signals generated by multi-source PDs with the use of the spark-gaps: (a) point-point and point-plane, (b) point-point and surface, (c) point-plane and surface.

\section{- point-plane system $(20-100 \mathrm{kHz})$,}

- surface system $(20-500 \mathrm{kHz})$.

Determination of the dominant frequencies corresponding to single-source discharges made it possible to attempt identification of basic PD forms in the case of multi-source discharge generation.

Analyzing the time-frequency images corresponding to multisource discharges there can be observed structures characteristic of varied frequency bands. These bans may be connected with dominant frequency ranges determined for basic PD forms. Hence it can be claimed that it is possible to recognize basic $\mathrm{PD}$ forms during partial discharge generation.

\section{Summary}

Diagnostics of electric power transformers is based on many measurement methods, which jointly provide complete information on the technical condition of a given unit. The acoustic emission method, which has been supplemented by the tools for the time-frequency analysis, has been used more and more often, especially in unit transformers. The research carried out proved that detection of multi-source discharges is possible thanks to 
the application of the joint time-frequency analysis. The investigations presented were carried out in laboratory conditions, in a tub bereft of the elements introducing additional suppression, such as: the core, windings etc. It seems vital that additional measurements should be taken in actual conditions because the occurring interference level may significantly hinder interpretation of measurement results.

\section{References}

[1] T. Boczar, A. Cichon, S. Borucki, in: Proc. Int. Conf. Acoustics'08 Paris (Euronoise), 2008, p. 2205.

[2] T. Boczar, S. Borucki, A. Cichon, in: Proc. Europ. Symp. of Acoustics - Acustica'08, Coimbra (Portugal) 2008, p. 1.

[3] T. Boczar, S. Borucki, A. Cichon, M. Lorenc, Arch. Acoust. 31 (Suppl.), 325 (2006).
[4] E. Gulski, Diagnostics Partial Discharges on the High Voltage Plant in Exploitation, Oficyna Politechniki Warszawskiej, Warszawa 2003 (in Polish).

[5] F. Witos, Z. Gacek, Z. Opilski, Acta Phys. Pol. A 114, A-249 (2008).

[6] T. Boczar, S. Borucki, A. Cichon, M. Lorenc, Acta Phys. Pol. A 114, A-21 (2008).

[7] F. Witos, Z. Gacek, P. Paduch, Mol. Quant. Acoust. 28, 271 (2007).

[8] T. Boczar, M. Szmechta, D. Zmarzły, Mol. Quant. Acoust. 28, 39 (2007).

[9] A. Szpakowski, C. Tyszkiewicz, T. Pustelny, Acta Phys. Pol. A 114, A-237 (2008).

[10] T. Boczar, S. Borucki, A. Cichon, Mol. Quant. Acoust. 28, 13 (2007). 